\title{
The prevalence of potentially inappropriate medication prescribing in elderly patients with chronic kidney disease
}

\author{
Sarah Amy Jones, ${ }^{1}$ Sunil Bhandari ${ }^{2}$
}

${ }^{1}$ York Teaching Hospital NHS Foundation Trust, York, UK ${ }^{2}$ Department of Nephrology, Hull and East Yorkshire Hospitals NHS Trust and Hull York Medical School, Hull, UK

\section{Correspondence to} Professor Sunil Bhandari, Department of Nephrology, Hull and East Yorkshire Hospitals NHS Trust and Hull York Medical School, Hull, UK; sunil.bhandari@hey.nhs.uk

Received 4 March 2012 Revised 6 January 2013 Accepted 22 January 2013 Published Online First 16 February 2013

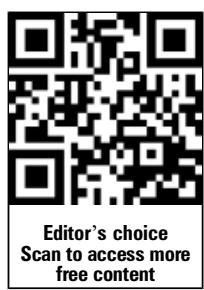

\section{SLinked}

- http://dx.doi.org/10.1136/ postgradmedj-2013-131764

To cite: Jones $S A$, Bhandari S. Postgrad Med J 2013;89:247-250.

\begin{abstract}
Introduction Potentially inappropriate medication (PIM) prescribing is a global problem. Limited data are available on the prevalence of PIMs in elderly patients with chronic kidney disease (CKD). We examined the PIM prevalence in elderly patients with CKD, the most common drugs implicated and the levels of

polypharmacy in this group.

Methods A retrospective case record analysis of patients with CKD above the age of 70 years was carried out on 100 consecutive inpatients between January 2008 and June 2008 at a University Teaching Hospital. PIMs were defined using the modified Beers' criteria and latest British National Formulary (BNF) guidance for prescribing in patients with renal impairment using creatinine clearance calculated by the Cockroft-Gault formula.
\end{abstract}

Results The mean age of the cohort was 80 years with a female predominance (62\%). The mean estimated glomerular filtration rate (eGFR) was $17.2 \mathrm{ml} / \mathrm{min} /$ $1.73 \mathrm{~m}^{2} .56$ patients had one or more PIMs prescribed during the acute admission period. 81 out of the 622 medications prescribed were 'inappropriate'; therefore, the prevalence of PIMs prescribed was $13 \%$. Antibiotics and antihypertensives accounted for the majority of PIMs. Each patient received an average of six drugs. Conclusions The prevalence of PIMs in elderly inpatients with CKD is high. Inclusion of drugs prescribed acutely and use of the BNF to identify PIMs as well as the Beers' criteria may account for this. Use of screening tools may lead to better identification of PIMs in this high risk group. Routine calculation and documentation of eGFR on the medication chart on admission may help reduce PIMs.

\section{INTRODUCTION}

Drug toxicity is a significant problem in the elderly. ${ }^{1}$ There is reduced excretion of drugs and their metabolites from kidneys impaired by disease and age related changes. There is a threefold greater incidence of adverse drug reactions (ADRs) in patients over 60 years of age compared with those under 30 years of age. ${ }^{2}$ ADRs are implicated in $5 \%-17 \%$ of hospital admissions. ${ }^{3}$

The prescribing of potentially inappropriate medications (PIMs) can lead to ADRs and result in increased morbidity, mortality and resource utilisation. ${ }^{4}$ Medications can be 'inappropriate' in the elderly because they are either ineffective or they pose unnecessary risk and a safer alternative is available. 'Inappropriateness' may also be due to the use of drugs contraindicated by a specific medical condition affecting the patient in question. $^{5}$

PIM prescribing is an increasing global healthcare problem. ${ }^{6}$ A study of elderly hospitalised patients in Europe found that $30.4 \%$ of patients were prescribed at least one PIM. However, this varied from 22.7\% in Prague to $43.3 \%$ in Geneva. ${ }^{7}$ The prevalence of elderly patients with moderate to severe renal impairment discharged from a UK hospital on at least one PIM has been quoted at $24 \%{ }^{1}$

Risk factors for the prescribing of PIMs include nursing home residential status, female sex, multiple illnesses, ${ }^{8}$ age below 85 years and polypharmacy. ${ }^{9}$ Of those aged 75 years or more, $36 \%$ take four or more clinician prescribed drugs regularly. ${ }^{10}$ Mortality and morbidity can be prevented in this group of patients by closely analysing and correcting prescribing practices. ${ }^{3}$ The National Service Framework for Older People in England and Wales recommends that all people above the age of 75 years should have their medications reviewed annually to help reduce the risks. For those taking four or more medications this should be at least six monthly. ${ }^{11}$

Various tools have been developed to identify PIMs. The Beers' criteria list medications to be avoided in the elderly. ${ }^{5}$ They were devised in 1991 for use in nursing home residents. A validated consensus technique involving several geriatric pharmacotherapy experts was used to agree on the original list of drugs. Since then the listed medications have been updated and it now gives two separate lists: one list 'considering diagnosis' and the other 'independent of diagnosis'.

In this study, we aimed to ascertain the prevalence rate of PIMs among elderly patients with renal impairment admitted to Hull Royal Infirmary, a tertiary teaching hospital. We aimed to identify the most common medications implicated in PIM prescriptions and to study the prevalence of polypharmacy in this group.

\section{METHODS}

\section{Inclusion criteria}

We defined elderly patients as those over 70 years of age. For the purpose of this study, patients with chronic kidney disease were those with CKD stages 3-5 based on the medical records and previous biochemistry. The estimated glomerular filtration rate (eGFR) was calculated by the 4-point modified diet in renal disease (MDRD) formula for each patient based on a creatinine level 3 months prior to admission to ensure they fitted the criteria for CKD 
3-5 (ie, an eGFR of less than $60 \mathrm{ml} / \mathrm{min} / 1.73 \mathrm{~m}^{2}$ ). This method was chosen based on the recognised national guidelines for the diagnosis of CKD. ${ }^{12}$

\section{Sample selection}

We selected 100 patients meeting the above criteria admitted to the medical admissions unit of a University Teaching Hospital (Hull Royal Infirmary) for any medical reason. Hull Royal Infirmary is part of Hull and East Yorkshire NHS Trust, one of the largest acute trusts in England. It provides services to 600000 people in the Hull and East Yorkshire region and 1.2 million in the wider region as a tertiary centre for specialties such as nephrology. A consecutive list of inpatients admitted between January 2008 and June 2008 was obtained and case notes were retrospectively analysed.

\section{Identification of PIMs}

PIMs were defined by using the modified Beers' criteria independent of diagnosis, modified Beers' criteria considering diagnosis $^{5}$ and the latest British National Formulary (BNF) guidance for prescribing in patients with renal impairment. At the study hospital, eGFR is not routinely reported for acute admissions by the lab unless requested. The eGFR used for the study was calculated based on the serum creatinine on admission. The eGFR measurements as per the Cockroft-Gault formula were calculated as recommended by the BNF for drug dose adjustments in renal impartment.

Using these criteria, a medication was deemed inappropriate by the authors if it was contraindicated or prescribed at an inappropriate dose for the level of renal function. All drugs listed on the standard hospital paper inpatient medication chart written up on admission including as required medication pro re nata (PRN) and once only medications were scrutinised. Intravenous fluid charts were not analysed in this study.

\section{Data analysis}

Patients' level of renal function, age, weight, sex and past medical history were recorded. All drugs and doses from the paper inpatient medication chart prescribed on admission (including 'once only' and 'as required' medications) were noted. Each drug's class was specified using the latest BNF to identify drug classes most commonly implicated as PIMs. In order to study the prevalence of polypharmacy, the total number of drugs prescribed to all the patients was noted and then the mean number of drugs prescribed to each patient was calculated. We analysed the number of patients receiving at least one PIM (PIM prevalence among patients) and the number of PIMs out of all medications prescribed (PIM prevalence among prescribed medications).

\section{Statistics}

Data are presented as the mean and range where specified. Significance testing was not performed.

\section{Ethical approval and confidentiality}

Ethical approval was not required to collect the data. The project was approved locally by the Hull Royal Infirmary Clinical Audit and Effectiveness Team. Confidentiality was maintained by using an anonymised database which was password protected and maintained on an encrypted computer in accordance with Trust guidelines. The only personal information collected for each patient was gender and date of birth.

\section{RESULTS}

\section{Patient demographics}

Of the 100 patients, the average age was 80 years (range 70 98 years). In all, $40 \%$ of patients were older than 80 years. Women accounted for $62 \%$ of the sample and made up 53\% of those above 80 years of age (figure 1 ).

\section{Kidney function}

The mean MDRD based eGFR was $17.2 \mathrm{ml} / \mathrm{min} / 1.73 \mathrm{~m}^{2}$ (range $4-44 \mathrm{ml} / \mathrm{min} / 1.73 \mathrm{~m}^{2}$ ). The mean eGFR calculated by the Cockroft-Gault formula was $17.3 \mathrm{ml} / \mathrm{min} / 1.73 \mathrm{~m}^{2}$ (range $3.2-$ $51.6 \mathrm{ml} / \mathrm{min} / 1.73 \mathrm{~m}^{2}$ ).

\section{PIMs}

A total of 56 out of the 100 patients had a PIM prescribed (ie, the prevalence rate of PIMs among patients was 56\%). A total of 622 medications were prescribed among the 100 patients with an average six medications per patient (range 1-12). Overall, $13 \%(81 / 622)$ of all medications prescribed were potentially inappropriate.

\section{Medications}

In all, 4\% (24/622) of prescriptions were for antibiotics. Antibiotics accounted for 21\% (17/81) of the total PIMs representing the largest category (figure 2). Vancomycin, penicillins and sulfonamides were the most common prescriptions.

Antihypertensive agents accounted for the next largest category of PIMs. Overall, 11\% (68/622) of prescriptions were for antihypertensives. Of the PIMs, 19\% (15/81) were antihypertensives. ACE inhibitors and angiotensin receptor blockers were most commonly implicated.

Overall, 2.6\% (16/622) of prescriptions were for antidiabetic drugs. Antidiabetic medications accounted for 15\% (12/81) of PIMs. Analgesic drugs accounted for $11 \%$ of PIMs (9/81). Tramadol, non-steroidal anti-inflammatory drugs (NSAIDs) and codeine based analgesics were commonly involved.

A variety of other drugs accounted for the remaining PIMs. These included diuretics $(7 \%, 6 / 81)$, heart rate limiting agents such as $\beta$ blockers and digoxin $(7 \%, 6 / 81)$ and lipid lowering agents (statins/fibrates) (4\%, 3/81).

\section{DISCUSSION}

Our study estimates the prevalence of PIMs in elderly patients with CKD in the tertiary setting. We found that more than half

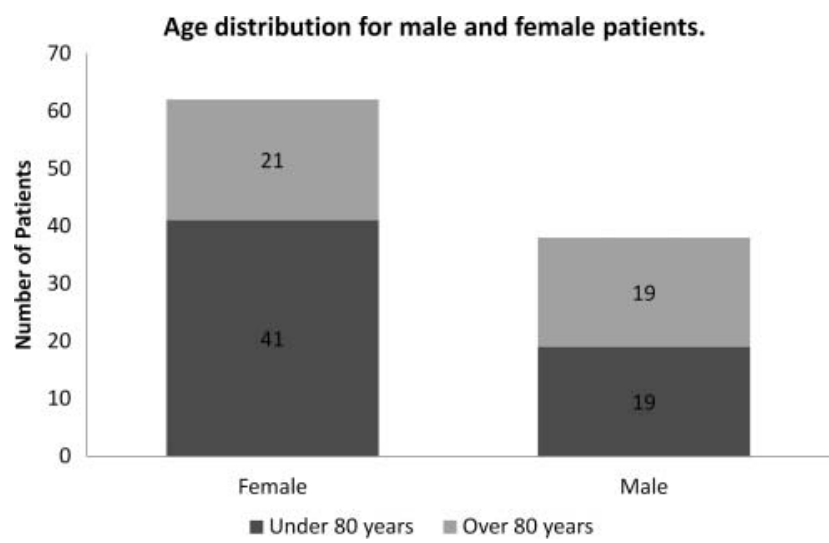

Figure 1 A bar graph showing the split of the number of male and female patients according to age of greater than 80 years versus 80 years or lower. 
Figure 2 A pie chart showing the percentage of the 81 potentially inappropriate medications (PIMs) from each drug class. CKD, chronic kidney disease.

\section{The percentage distribution of PIMs amongst the prescriptions of elderly patients with CKD.}

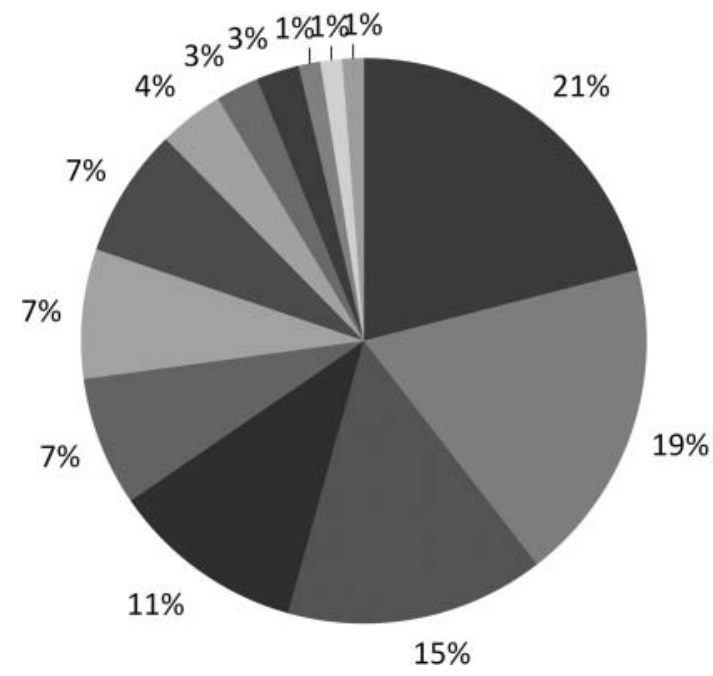

of patients in our sample were prescribed at least one medication that was potentially inappropriate. Of all medications prescribed, $13 \%$ were identified as PIMs.

A number of studies have looked at the prevalence of PIMs and found lower prevalence rates than our study. Figures of $30 \%,{ }^{7} 34 \%{ }^{13}$ and $32 \%{ }^{4}$ for PIM prevalence among patients have been quoted in UK studies using solely the Beers' criteria to analyse the medications patients were taking immediately prior to acute admission (excluding drugs prescribed after admission). American studies using the Beers' criteria to identify PIMs found that $12.6 \%{ }^{14}$ and $6.5 \%{ }^{15}$ of emergency department attendees were prescribed at least one PIM while in the emergency department. One Oxfordshire study found that $24 \%$ of patients with moderate to severe renal impairment were prescribed at least one PIM on discharge from hospital. ${ }^{1}$ Previous studies have found the prevalence of PIMs among all prescribed medications to be $7.6 \%,{ }^{4} 8.2 \%{ }^{13}$ and $3 \% .{ }^{16}$ Again, these figures are lower than in the present study but they did not include acutely prescribed medications or PIMs identified using the BNF.

The most common classes of PIMs differed in our study to previous studies. Benzodiazepines and tricyclic antidepressants used in patients with frequent falls, ${ }^{7}$ NSAIDs and opiates ${ }^{17}$ were the most commonly identified PIMs in recent previous studies. Polypharmacy levels were similar; an average of $4.5,{ }^{16} 5^{4}$ and $6^{17}$ medications per patient were quoted in previous studies.

Our PIM prevalence among patients is higher than that quoted in the literature. This may be for a number of reasons. Our sample includes patients with CKD who often require dose adjustments, especially for antibiotics which were the most commonly identified PIM in our sample. Our medication data include those drugs prescribed acutely. No other study to our knowledge has examined PIMs in elderly inpatients with CKD including medications prescribed acutely. As well as the Beers' criteria we have used the BNF to identify PIMs which has not been done in other studies. This has led to us identifying more PIM classes than if we had used the Beers' criteria alone and perhaps explains why the most common classes of PIMs differ.

The Beers' criteria were chosen for use in this study as they are a long standing, well-validated tool which makes our results easily comparable with other studies. There are many criticisms of the Beers' criteria. It is a tool designed for use in USA; therefore, many of the medications on its list are not available in Europe. ${ }^{18}$ It omits many commonly used drugs in Europe and does not address drug-drug interactions, drug duplications or drug omissions ${ }^{19}$ which highlights issues of its applicability in a UK setting.

There are alternatives to the Beers' criteria such as the Improved Prescribing in the Elderly Tool (IPET) ${ }^{13}$ and the Screening Tool of Older Persons' Potentially Inappropriate Prescriptions (STOPP). ${ }^{20}$ Unlike the IPET and Beers' criteria, STOPP is organised in a logical manner, includes drug omissions and has taken steps to be internationally applicable. There is no prescribing appropriateness tool to our knowledge specific for elderly inpatients with CKD.

One aspect which was not assessed in the current study was the lack of a unified formula which can be used both for calculating the stage of CKD and for prescribing in renal impairment. CKD stages are classified based on eGFR levels calculated by the 4-point MDRD formula, while prescribing as per the BNF is based on creatinine clearance calculated by the Cockroft-Gault formula. This is an area which perhaps deserves further study as confusion around this issue ${ }^{21}$ may be a possible reason for lack of assessment of renal function.

Commonly prescribed medications such as antibiotics and antihypertensives account for the majority of PIMs in elderly inpatients with CKD admitted acutely to a tertiary hospital. Given the frequency with which these medications are prescribed in the wider community, it is important that care is taken to ensure appropriate doses are prescribed in relation to the level of renal impairment. The routine measurement and documentation of eGFR for elderly patients on admission may serve to remind clinicians of impaired renal function despite a relatively normal serum creatinine concentration. Awareness of abnormal renal function affecting acute admissions and education about common antibiotics that require dose adjustments may help to reduce PIM prescribing.

This study was small and confined to a single tertiary hospital setting thus creating room for selection bias. A larger study over a wider area is perhaps required in order to validate our findings. The use of newer tools such as the STOPP or a purpose designed tool for elderly patients with renal impairment may lead to better identification of PIMs in this high risk group. We have been unable to calculate the proportion of PIMs due to 
wrong drug as opposed to wrong dose and the proportion of PIMs identified by the BNF rather than the Beer's criteria in this study. This information would be of interest in future studies. Data on the grade of doctors responsible for prescribing PIMs and the presenting complaints of patients prescribed PIMs were not collected in this study. It would be useful to investigate these areas to help target education of doctors and highlight patient groups at particular risk.

\section{Main messages}

- The prevalence of potentially inappropriate medication (PIM) prescribing during acute admission is high among elderly patients with renal impairment.

- Commonly prescribed drugs such as antibiotics and antihypertensives account for the majority of PIMs.

- Polypharmacy is highly prevalent among elderly patients with renal impairment.

\section{Current research questions}

- Are the findings in this study true of larger populations and in different areas of the country?

- Would routine calculation and documentation of eGFR on admission reduce the number of potentially inappropriate medications (PIMs) prescribed for elderly patients with chronic kidney disease (CKD) in the acute setting?

- Which doctors are likely to prescribe PIMs and why? What steps can be taken to reduce this?

- Which types of patient with CKD are particularly likely to be prescribed PIMs?

- Should elderly patients with CKD have more regular checks of their medications to help reduce the prevalence of PIMs, perhaps using tools such as the Screening Tool of Older Persons' Potentially Inappropriate Prescriptions, or a purpose built tool?

\section{Key references}

- Gallagher P, Lang PO, Cherubini A, et al. Prevalence of potentially inappropriate prescribing in an acutely ill population of older patients admitted to six European hospitals. Eur J Clin Pharmacol 2011;67:1175-88.

- MacGregor MS, Taal MW. Renal Association Clinical Practice Guideline on detection, monitoring and management of patients with CKD. Nephron Clin Pract Published Online First: 6 May 2011. doi=10.1159/ 000328062\&typ

- Barry PJ, O'Keefe N, O'Connor KA, et al. Inappropriate prescribing in the elderly: a comparison of the Beers criteria and the improved prescribing in the elderly tool (IPET) in acutely ill elderly hospitalised patients. J Clin Pharm Ther 2006;31:617-26.

- Stevens LA, Coresh J, Greene T, et al. Assessing kidney function-measured and estimated glomerular filtration rate. N Engl J Med 2006;354:2473-83.
Acknowledgements We would like to thank Dr Anoop Amarnath for data collection.

Contributors SB devised and oversaw the study, analysed and interpreted the data and edited the various versions. SAJ revised and restructured the initial draft and figures and checked analysis of the data.

Competing interests Both authors have completed the Unified Competing Interest form at http://www.icmje.org/coi disclosure.pdf (available on request from the corresponding author). SAJ and SB have no competing interests. SB has accepted honoraria for lectures and grants towards attendance of the American Transplant Congress and American Society of Nephrology from Amgen, Pharmacosmos, Pfizer, Novartis, Genzyme and Shire. SB has been a primary investigator in several pharmaceutical studies funded by Amgen, Pharmacosmos, Vifor and Roche.

Provenance and peer review Not commissioned; externally peer reviewed.

\section{REFERENCES}

1 Wong NA, Jones HW. An analysis of discharge drug prescribing amongst elderly patients with renal impairment. Postgrad Med J 1998;74:420-2.

2 Denham MJ, Barnett NL. Drug therapy and the older person. Role of the pharmacist. Drug Saf 1998;19:243-50.

3 Department of Health. Medicines and Older People_-Implementing medicines-related aspects of the NSF for Older People, 23634. March 2001.

4 Gallagher PF, Barry PJ, Ryan C, et al. Inappropriate prescribing in an acutely ill population of elderly patients as determined by Beers' Criteria. Age Ageing 2008;37:96-101.

5 Fick DM, Cooper JW, Wade WE, et al. Updating the Beers criteria for potentially inappropriate medication use in older adults. Arch Intern Med 2003; 163:2716-24.

6 Van Der Hooft CS, Jong GW, Dieleman JP, et al. Inappropriate drug prescribing in older adults: the updated 2002 Beers criteria-a population based cohort study. $\mathrm{Br}$ J Clin Pharmacol 2005;60:137-44.

7 Gallagher P, Lang PO, Cherubini A, et al. Prevalence of potentially inappropriate prescribing in an acutely ill population of older patients admitted to six European hospitals. Eur J Clin Pharmacol 2011;67:1175-88.

8 Gallagher P, Barry P, O'Mahony D. Inappropriate prescribing in the elderly. J Clin Pharm Ther 2007:32:113-21.

9 Fialova D, Topinkova E, Gambassi G, et al. Potentially inappropriate medication use among elderly home care patients in Europe. JAMA 2005;293;1348-58.

10 Health Survey for England. Cardiovascular Disease Volume 1 The Stationary Office (Online), 1998, http://www.archive.official-documents.co.uk/document/doh/survey98/ hse98.htm (accessed 24 Sep 2012).

11 Department of Health. National Service Framework for Older People, 23633. May 2001.

12 MacGregor MS, Taal MW. Renal Association Clinical Practice Guideline on detection, monitoring and management of patients with CKD. Nephron Clin Pract Published Online First: 6 May 2011. doi=10.1159/000328062\&typ

13 Barry PJ, O'Keefe N, O'Connor KA, et al. Inappropriate prescribing in the elderly: a comparison of the Beers criteria and the improved prescribing in the elderly too (IPET) in acutely ill elderly hospitalised patients. J Clin Pharm Ther 2006:31:617-26.

14 Caterino JM, Emond JA, Camargo CA. Inappropriate medication administration to the acutely ill elderly: a nationwide emergency department study, 1992-2000. JAGS 2004:52;1847-55.

15 Nixdorff N, Hustey FM, Brady AK, et al. Potentially inappropriate medications and adverse drug effects in elders in the ED. Am J Emerg Med 2008:26;697-700.

16 Ryan C, O'Mahony D, Kennedy J, et al. Appropriate prescribing in the elderly: an investigation of two screening tools, Beers criteria considering diagnosis and independent of diagnosis and improved prescribing in the elderly tool to identify inappropriate use of medicines in the elderly in primary care in Ireland. J Clin Pharm Ther 2009:34;369-76.

17 Gallagher P, O'Mahony D. STOPP (Screening Tool of Older Persons' potentially inappropriate Prescriptions): application to acutely ill elderly patients and comparison with Beers' criteria. Age Ageing 2008:37;673-9.

18 Dimitrow MS, Airaksinen MS, Kivela $S$, et al. Comparison of prescribing criteria to evaluate appropriateness of drug treatment in individuals aged 65 and older: a systematic review. J Am Geriatr Soc 2011;59:1521-30.

19 Levy HB, Marcus E, Christen C. Beyond the Beers criteria: a comparative overview of explicit criteria. Ann Pharmacother 2010:44;1968-75

20 Gallagher P, Ryan C, Byrne S, et al. STOPP (Screening Tool of Older Person's Prescription) and START (Screening Tool to Alert doctors to Right Treatment). Consensus validation. Int J Clin Pharmacol Ther 2008:46:72-83.

21 Stevens LA, Coresh J, Greene T, et al. Assessing kidney function-measured and estimated glomerular filtration rate. N Engl J Med 2006;354:2473-83. 